${ }^{5}$ Radcliffe Institute of Advanced

Study, Harvard University, Cambridge,

Massachusetts 02138, USA.
Present address: Laboratorios de

Investigación y Desarrollo, Facultad de

Ciencias y Filosofía, Universidad Peruana
Cayetano Heredia, Lima, Peru.

*e-mail: petros@ethz.ch

These authors contributed equally to this work.

\title{
Reply to 'On phonons and water flow enhancement in carbon nanotubes'
}

Ma et al. reply - We are glad to see that Cruz-Chú et al. ${ }^{1}$ followed our work showing the importance of coupling between the motion of water molecules confined in double-walled carbon nanotubes and the longitudinal phonon modes of the nanotubes. In agreement with our simulations, they found an enhancement of diffusion of confined water and oscillation in the shear stress induced by the coupling with phonons. However, using a different model for water, they observed a smaller enhancement of the diffusion coefficient of the water slab than that predicted in our work. In addition, they showed that the magnitude of enhancement depends on the choice of reference phononfree system.

In our original work ${ }^{2}$, the diffusion coefficient of the centre of mass of water confined in double-walled carbon nanotubes was calculated using two models of water, the coarse-grained model $(\mathrm{mW})$ and the all-atom model (TIP4P/2005), with the same methodology as in the correspondence by Cruz-Chú et al. It should be noted that the TIP4P/2005 model reproduces water properties no worse than the TIP4P-Ew model used by Cruz-Chú et al., as demonstrated in ref. 3. Our simulations show that both models predict similar enhancement of the diffusion coefficient $D_{\mathrm{ac}}$ for the phononactive over the phonon-free nanotube (Fig. 1).

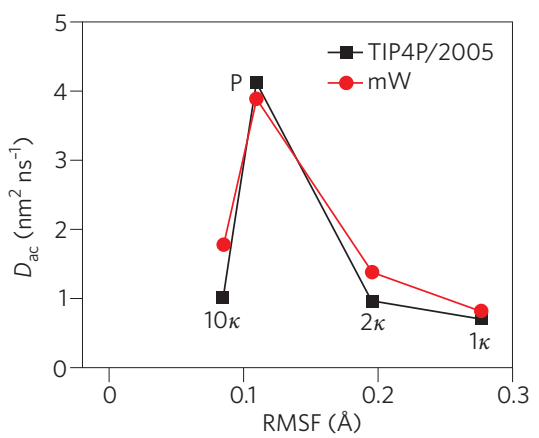

Figure 1 | Diffusion coefficient of water slab. Black squares and red filled circles correspond to coefficients calculated using the TIP4P/2005 and $\mathrm{mW}$ water models, respectively. The $\mathrm{P}$ label refers to phonon-active cases, and $1 \kappa, 2 \kappa$ and $10 \kappa$ labels refer to phonon-free cases with different spring constants. The double-walled carbon nanotubes are ordered according to the rootmean-square fluctuations (RMSF) of the atoms of the inner nanotube.
There is a slight dependence of the diffusion coefficient calculated for the reference phonon-free system on the choice of the stiffness of the spring connecting the carbon atoms with their equilibrium positions. However, for all values of parameters, we found more than $200 \%$ enhancement of the diffusion coefficients.

The coupling between the phonon modes and water can be understood by considering the effect of surface oscillations on interfacial friction ${ }^{2}$. This conclusion has been supported by the calculations of friction coefficient carried out by Cruz-Chú et al., which demonstrated a reduction of interfacial friction due to surface oscillation. This is consistent with our previous predictions of oscillationinduced reduction of interfacial friction and corresponding enhancement of diffusion, which have been done within a generalized Prandtl-Tomlinson model for the interfacial dynamics ${ }^{4}$. We would like to emphasize that both effects have the same physical origin rather than being mutually exclusive.

Besides calculating the diffusion coefficient of the centre of mass of confined water, $D_{\mathrm{ac}}$, which was discussed in our work ${ }^{2}$, Cruz-Chú et al. also considered the diffusion coefficients of oxygen atoms, $D_{\mathrm{O}}$, and the effect of coupling with phonons on this quantity. This is an interesting characteristic of the confined fluid, which we did not study. But its relation to $D_{\mathrm{ac}}$ is not straightforward. In particular, $D_{\mathrm{ac}}$ is determined not only by diffusion of individual water molecules but also by correlations between the motion of different molecules. This can be clearly inferred by expanding (equation (1) in the work of Cruz-Chú et al.) for both $D_{\mathrm{ac}}$ and $D_{\mathrm{O}}$, and setting $N_{\mathrm{D}}$ to be 1 , that is, only studying the diffusion along the axial direction. As a result, the coupling of water motion with phonons may have a different effect on $D_{\mathrm{ac}}$ and $D_{\mathrm{O}}$, as found by Cruz-Chú and colleagues.

Our simulations show that the predicted enhancement of diffusion of confined water due to the coupling with the longitudinal phonon modes of the nanotubes depends only marginally on the model of water and on the choice of the phonon-free reference system. The enhancement of diffusion is directly related to phonon-induced reduction of friction: these are two facets of the same phenomenon.
Data availability

The data that support the plots within this paper and other findings of this study are available from the corresponding author upon reasonable request.

References

1. Cruz-Chú, E. R. et al. Nat. Nanotech. 12, 1106-1108 (2017)

2. Ma, M. et al. Nat. Nanotech. 10, 692-695 (2015).

3. Abascal, J. L. F. \& Vega, C. J. Chem. Phys. 123, 234505 (2005).

4. Tshiprut, Z., Filippov, A. E. \& Urbakh, M. Phys. Rev. Lett. 95, 016101 (2005)

Additional information

Reprints and permission information is available online at www.nature.com/reprints. Correspondence and requests for materials should be addressed to F.G. and Q.Z.

Competing financial interests

The authors declare no competing financial interests.

Ming Ma1,2,3*, Francois Grey ${ }^{3,4,5,6 \star}$ Luming Shen ${ }^{7}$, Michael Urbakh ${ }^{8,9}$, Shuai $\mathrm{Wu}^{3,10}$, Jefferson Zhe Liu"1 ${ }^{11}$ Yilun Liu' and Quanshui Zheng ${ }^{1,3,9,10,13 \star}$

'State Key Laboratory of Tribology, Tsinghua University, Beijing 100084, China.

${ }^{2}$ Department of Mechanical Engineering, Tsinghua University, Beijing 100084, China. ${ }^{3}$ Center for Nano and Micro Mechanics, Tsinghua University, Beijing 100084, China, ${ }^{4}$ London Centre for Nanotechnology, University College London, London WC1H OAJ, UK.

${ }^{5}$ Department of Physics, Tsinghua University, Beijing 100084, China.

${ }^{6}$ Citizen Cyberlab, CUI, University of Geneva, $\mathrm{CH}-1227$ Carouge, Switzerland.

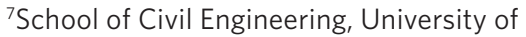
Sydney, Sydney, NSW 2006, Australia.

${ }^{8}$ School of Chemistry, Tel Aviv University, 69978 Tel Aviv, Israel.

${ }^{9}$ XIN Center, Tsinghua University, Beijing 100084, China, and Tel Aviv University, 69978 Tel Aviv, Israel.

${ }^{10}$ Department of Engineering Mechanics, Tsinghua University, Beijing 100084, China.

"Department of Mechanical Engineering,

The University of Melbourne, Melbourne, VIC 3010, Australia.

${ }^{12}$ International Center for Applied Mechanics, SV Lab, School of Aerospace, Xi'an Jiaotong University, Xi'an 710049, China.

${ }^{13}$ Applied Mechanics Lab, Tsinghua University، Beijing 100084, China.

*email: maming16@tsinghua.edu.cn;

francois.grey@unige.ch;

zhengqs@tsinghua.edu.cn 